\title{
d-11. A Device for Cerebrospinal Fluid Cytology of the Glioma
}

\author{
Yutaka Inaba, Umeo Ito, Hideo Hiratsuka, Akira Kamisasa and \\ Kiyohide Komatsu \\ Department of Neurosurgery, School of Medicine, Tokyo Medical and Dental University
}

Diagnostic cytology of the cerebrospinal fluid is not fully established nowadays. The reasons are: 1) It is difficult to collect cells, because cell number is very limited in the CSF. 2) The tumor cell does not always appear in the CSF of patient with glioma. 3) When the glioma cells appear, they are very scanty in numbr. 4) The procedure in making diagnosis of the glioma cells are still in confusion, now. 5) Morphological criteria for the diagnosis of tumor kind and malignancy of the glioma cells are not fully established.

In order to collect the CSF cells effectively and without causing morphological dainage to the cells, many kinds of attempt had been made. Recently, Kolar and Zeman (1968) constructed and apparatus reforming Sayk's sedimentation chamber method, and which provided cytomorphologic preparation of good quality in the small circle on the glass slide. However, from $20-90 \%$ of the CSF cells are lost in the filter paper. Then we deviced an apparatus of centrifugating sedimentation chamber itself, to make CSF stream in the chamber straight to the slide glass and increase the gravitation vector on the cells to $100-500 \mathrm{G}$. We succeeded in increasing the collected cell number about 20-40 times than that using Kolar's apparatus. There is not any significant qualitative change in the cells caused by increased $\mathrm{G}$.

Cytological diagnosis of the CSF glioma cells can not be carried out according to Papanicoloau's criteria. Because, 1) not all of the glioma are malignant as is said in other organs, 2) when the glioma is malignant in character, morphological malignancy of the cells is lower than that of other organs. We make the diagnosis of CSF glioma cells following the procedure described below: Investigate as many normally and reactively appearing cells as possible, and then find out unusual cells which seem to be without the limit of reactively appearing cells; estimate the possibility of brain tumor on the basis of percent rate of the appeared unusual cells; if possible, make diagnosis of tumor kind and malignancy, following the morphological specificity of the cells.

We succeeded in obtaining electron-microscopic picture of the CSF cells, and found some good informations, comparing the picture fo operation material from the same patient, in making the diagnosis of the glioma cells. Also, we succeeded in labeling the malignant glioma cells in histoautoradiographic preparation, incubating the CSF by ${ }^{3} \mathrm{H}$-Thymidine, in vitro. It seems probable that this technique contribute to determining the malignancy of the glioma cells appeared in the CSF. 